\title{
Dissolving foreign bodies in the trachea and bronchus
}

\author{
A. J. ME A R N S a n R . M. E N G L A N D \\ Paediatric Cardio-Thoracic Unit, Harefield Hospital and ENT Unit, \\ Hillingdon Hospital, Middx
}

\begin{abstract}
Mearns, A. J. and England, R. M. (1975). Thorax, 30, 461-463. Dissolving foreign bodies in the trachea and bronchus. Two young children inhaled sweets which dissolved in the tracheobronchial secretions and caused severe respiratory obstruction. The viscid fluid produced as the sweet dissolved was not expectorated and bronchoscopy was necessary. The oedema of the mucosa caused by the presence of a hyperosmolar sugar solution took up to 48 hours to resolve; antibiotics and steroid cover were considered necessary. The similarity of presentation with acute tracheobronchitis and the lack of reports in the literature suggest that such cases may be misdiagnosed.
\end{abstract}

\section{CASE REPORTS}

CASE 1 A boy aged 3 years and 6 months had been given a small packet of sweets. He had eaten these by tipping the contents into his mouth. When he coughed the mother realized that he had inhaled one or more of the sweets. The child was taken to the local casualty department as the mother had noticed increasing stridor. A radiograph of the chest was normal. Stridor was confirmed and the child was referred to the Paediatric Cardio-Thoracic Unit at Harefield Hospital. During this time stridor became more marked and the breathing increased in rate and effort, intercostal recession becoming obvious. We proceeded rapidly to bronchoscopy. Open ether induction anaesthesia was used. The sub-glottic area was oedematous, and pink viscous fluid obstructed the trachea immediately below the cords. This viscous fluid was removed with diffculty by suction, and the tracheal mucosa was found to be oedematous.

Stridor and respiratory difficulties persisted when the bronchoscope was removed, and a patent airway was obtained only by nasotracheal intubation. This tube was left in situ for 48 hours. The child was given intravenous hydrocortisone, $100 \mathrm{mg}$ sixhourly, and also ampicillin and cloxacillin. He was nursed in a maximum humidity oxygen tent until extubated on the second day. He soon returned to normal. Some rhonchi persisted for a few days but no parenchymatous lung disease developed. He was well at follow-up four months later.

CASE 2 A boy aged 22 months, with no previous history of respiratory disease, was a passenger on a plane leaving Heathrow Airport. He was eating a sweet during take-off when he coughed and went blue. The child rapidly developed stridor and became unconscious.

The plane returned at once to Heathrow and the child was transferred to Hillingdon Hospital by ambulance. During this acute period an air hostess applied mouth to mouth respiration. On admission the child was semi-conscious with slight cyanosis, tracheal stridor, and inspiratory intercostal recession. He showed incomplete signs of decerebrate rigidity with opisthotonos and jaw clenching. Secretions appeared in the larynx, requiring aspiration. The pulse rate was 96 per minute, sinus rhythm. There was equal air entry into both lungs.

Urgent bronchoscopy showed oedema of the tracheal mucosa with thick yellowish fluid in the trachea and both main bronchi.

The bronchial tree was aspirated. The child was then nursed in a maximum humidity oxygen tent with a pharyngeal airway in situ. The child was now pink and semi-conscious with continuing respiratory stridor. Betamethasone, $4 \mathrm{mg}$ statim then in reducing doses over three days, was given intravenously, with ampicillin and cloxacillin. Stridor subsided slowly. A further radiograph showed patchy atelectasis in both upper lobes which cleared in 24 hours. Over the next 48 hours the child regained consciousness and took oral fluids. The rigidity disappeared by the fifth day. The child developed an evening pyrexia and a rash which proved to be due to roseola infantum. He was discharged on the ninth day.

Six weeks later the child showed no residual neurological or respiratory deficit, and the mother considered the child was normal. 


\section{DISCUSSION}

It would seem from these case histories that when a sweet enters the trachea or bronchus and is not expectorated it dissolves slowly. The syrup formed around the sweet is a viscous, hyperosmolar solution which the child is unable to expectorate. This hyperosmolar solution draws fluid from the mucosa, causes local oedema, and reduces the lumen of the trachea and bronchi. Increasing stridor and respiratory effort are a measure of the developing obstruction.

In neither of our cases was the child able to expectorate. Even after bronchoscopy the mucosal oedema caused continuing respiratory obstruction. Airway patency was preserved by nasotracheal intubation in case 1 , by pharyngeal intubation in case 2 , and by steroids with antibiotic cover in both cases.

Case 1 was given a packet of sweets which he tipped into his mouth with the head held back. His initial symptoms were minimal, and only the slight changes in his breath sounds alerted the mother. The deterioration and difficulties encountered at bronchoscopy indicate that any suspicion that an inhaled foreign body is a sweet warrants urgent bronchoscopy.

Burrington and Cotton (1972) suggest that postural drainage and clapping of the back, with inhalation of isoprenaline, will remove $80 \%$ of foreign bodies. They also suggest that there is no indication for urgent bronchoscopy, and that it should be done only after a 24-hour trial of conservative methods. Jackson (1915) stated that soluble material such as candy, or foods which disintegrate quickly, need cause no uneasiness as they are soon coughed up.

When a sweet lodges in the larynx or trachea, although it may be expelled immediately, it may produce asphyxiation and death (Weston, 1965; Jackson and Jackson, 1945).

In case 2 it is likely that the sweet had been reduced in size by the effect of either sucking or chewing as its initial size would not have permitted passage into the trachea. There is little chance of finding any solid fragments of sweet by the time a bronchoscope is passed, unless bronchoscopy is carried out soon after the incident. Both the cases were bronchoscoped within four hours.

The clinical picture presented is similar to that of some cases of acute laryngotracheobronchitis. The endoscopic appearance of oedematous mucosa and thick secretions is consistent with this diagnosis. Indeed, initially in case 2 at bronchoscopy an acute infection seemed likely because of the nature of the secretions, the oedema and the absence of a foreign body. However, it was $\stackrel{\overrightarrow{\bar{O}}}{\stackrel{\vec{S}}{ }}$ obvious from the history that the child had in- $\bar{C}$ haled a sweet.

The appearance of the chest radiograph in case $\frac{\bar{\omega}}{\frac{5}{\sigma}}$ 2 , showing scattered atelectasis of both upper $\stackrel{\mathbb{Q}}{\AA}$ zones, was probably due to absorption collapse; 零 the viscid secretions could obstruct the bronchioles and then diffuse as the viscosity of the solution fell. This type of radiological appearance is also $\vec{\omega}$ seen in acute tracheobronchitis.

It is difficult to accept that the problem of $\vec{x}$ dissolving foreign todies in the bronchus has not been widely recognized. Nevertheless, in their detailed list of 3266 instances of foreign bodies in the air and food passages of patients of all ages, $\stackrel{\rightarrow}{\triangle}$ Jackson and Jackson (1936) make no mention of 을 dissolving sweets. This is also true of other series which include adults (Kassay, 1960; Brooks, 1972). The reports of a series of foreign bodies in the airways of children from Liverpool (Davis, 1966), 兵 Melbourne (Pyman, 1971), Denver (Burrington $\vec{\theta}$ and Cotton, 1972), and Toronto (Kim et al., 1973) जे do not specifically mention dissolving sweets.

The extent of oedema of the tracheobronchial wall is difficult to assess through an infant's bronchoscope. There may be other possibilities to $\bar{\partial}$ account for the events which followed the presumed inhalation. The history is important as it $\varnothing$ is possible that a child could cough up and swallow $\overrightarrow{\overrightarrow{0}}$ the sweet immediately after inhalation, making $\exists$ the course of the subsequent respiratory difficulty a less easily explained phenomenon. In both the cases recorded, however, the presence of intelli- $\Rightarrow$ gent observers verified the history. Although both children were given antibiotics there was no real evidence of any infection. White cell counts were $\frac{\text { O }}{3}$ not raised, and there was no pyrexia during the three days after bronchoscopy.

\section{CONCLUSION}

Endotracheal and bronchial foreign bodies in small children continue to present a clinical problem. These two cases illustrate an unusual aspect, $N$ when the foreign body has dissolved in the tracheobronchial secretions.

The practice of allowing a small child to tip a packet of sweets into the mouth is to be deplored.?

We wish to thank Dr. H. Finlay for advice during the preparation of the paper. Mr. J. W. Jackson has? kindly permitted publication of case 1 who was under 0 his care.

\section{REFERENCES}

Brooks, J. W. (1972). Foreign bodies in the air and food passages. Annals of Surgery, 175, 720. 
Burrington, J. D. and Cotton, E. K. (1972). Removal of foreign bodies from the tracheobronchial tree. Journal of Paediatric Surgery, 7, 119.

Davis, C. M. (1966). Inhaled foreign bodies in children. An analysis of 40 cases. Archives of Disease in Childhood, 41, 402.

Jackson, C. (1915). Peroral Endoscopy and Laryngeal Surgery, p. 237. Laryngoscope Company, St. Louis.

- and Jackson, C. L. (1936). Diseases of the Air and Food Passages of Foreign-body Origin. W. B. Saunders, Philadelphia and London. and - (1945). Diseases of the Nose, Throat and Ear including Bronchoscopy and Oesophagoscopy, p. 747. W. B. Saunders, Philadelphia and London.
Kassay, D. (1960). Observations on one hundred cases of bronchial foreign body. Archives of Otolaryngology, 71, 42.

Kim, I. G., Brummitt, W. M., Humphrey, A., Siomra, S. W., and Wallace, W. B. (1973). Foreign body in the airway: a review of 202 cases. Laryngoscope, 83, 347 .

Pyman, C. (1971). Inhaled foreign bodies in childhood. A review of 230 cases. Medical Journal of Australia, 1, 62.

Weston, J. T. (1965). Airway foreign body fatalities in children. Annals of Otology, Rhinology and Laryngology, 74, 1144.

Requests for reprints to: A. J. Mearns, FRCS, c/o Cardio-Thoracic Dept., Leeds General Infirmary, Leeds. 